\title{
Spin-wave propagation in the presence of inhomogeneous Dzyaloshinskii-Moriya interactions
}

\author{
Seung-Jae Lee, ${ }^{1}$ Jung-Hwan Moon, ${ }^{2}$ Hyun-Woo Lee, ${ }^{3}$ and Kyung-Jin Lee ${ }^{1,2, *}$ \\ ${ }^{1}$ KU-KIST Graduate School of Converging Science and Technology, Korea University, Seoul 02841, Korea \\ ${ }^{2}$ Department of Materials Science and Engineering, Korea University, Seoul 02841, Korea \\ ${ }^{3}$ PCTP and Department of Physics, Pohang University of Science and Technology, Pohang 37673, Korea \\ (Received 11 April 2017; revised manuscript received 30 October 2017; published 27 November 2017)
}

\begin{abstract}
We theoretically investigate spin-wave propagation through a magnetic metamaterial with spatially modulated Dzyaloshinskii-Moriya interaction. We establish an effective Schrödinger equation for spin waves and derive boundary conditions for spin waves passing through the boundary between two regions having different Dzyaloshinskii-Moriya interactions. Based on these boundary conditions, we find that the spin wave can be amplified at the boundary and the spin-wave band gap is tunable either by an external magnetic field or the strength of Dzyaloshinskii-Moriya interaction, which offers a spin-wave analog of the field-effect transistor in traditional electronics.
\end{abstract}

DOI: 10.1103/PhysRevB.96.184433

\section{INTRODUCTION}

The Dzyaloshinskii-Moriya interaction (DMI) is the antisymmetric component of quantum mechanical exchange interaction in magnetic systems [1,2]. Three prerequisites of the DMI are the exchange interaction, the spin-orbit interaction, and the inversion symmetry breaking. All these three prerequisites are simultaneously satisfied in material systems such as B20 structures [3-5] and ferromagnet/heavy metal bilayer structures [6-12]. The DMI affects the equilibrium spin texture and consequently magnetization dynamics by stabilizing chiral domain walls [13-16] or magnetic skyrmions [17-23]. The DMI also causes the nonreciprocal spin-wave propagation [24-26], which is widely used to estimate the strength of DMI [27-31]. Recent works found that the DMI effect on the spin-wave propagation can also result in unidirectional caustic beams [32], spin-wave diodes [33], and spin-wave fibers [34], opening rich spin-wave physics and wide applications in functional devices based on spin waves.

The magnonic crystal is a magnetic metamaterial with alternating magnetic properties that serve as periodic potential for spin waves passing through it [35-48]. As spin waves, the collective precessional motion of localized electron spins, do not involve the motion of electrons, magnonic devices avoid Joule heating and thus allow low-power computing $[36,38,46,47]$. Moreover, their wave properties provide distinct functionalities [49-53] such as multi-input/output (nonlinear) operations [54,55]. Despite their attractive features, however, magnonic devices suffer from a small on/off ratio of spin-wave signal. We note that in traditional electronic logic devices based on field-effect transistors, the source-drain current substantially varies by a gate voltage. For practical use of magnonic devices, therefore, it is of critical importance to largely modulate spin-wave signals for a given spin-wave frequency. For this purpose, a possible way is to modulate the spin-wave band gap by an external means; for a given spinwave frequency, opening/closing the band gap at the frequency offers a large change in the signal of propagating spin waves through a magnonic crystal. In this work, we theoretically

*kj_lee@korea.ac.kr demonstrate that magnonic crystals with alternating DMI show an efficient modulation of spin-wave signal. We consider the interfacial DMI present in ferromagnet/heavy metal bilayers but the working principle is also applicable to arbitrary DMI symmetries, including the bulk DMI in B20 structures, by rotating the $\mathrm{DM}$ vector.

We first focus on a magnetic thin film with a vanishing demagnetization effect, which is experimentally achievable by tuning the thickness of a thin film having the surface perpendicular anisotropy. In such thin film structures, a spatial modulation of interfacial DMI can be realized by a local gating [56], a local modulation of the interface between ferromagnetic layer and heavy metal layer [57-59], or a local variation of the heavy metal thickness [60]. For a magnonic crystal with spatially modulated DMI, we establish an effective Schrödinger equation for spin waves and derive spin-wave boundary conditions at the boundary between two regions having different DMI values. With these boundary conditions, we construct a spin-wave version of the Kronig-Penny model [61], which is a simplified model for an electron in a onedimensional periodic potential. At the end of this paper, we show that our finding, an efficient tunability of spin-wave band gap in DMI-modulated magnonic crystals, also holds for magnetic thin films with a finite demagnetization effect.

\section{ANALYTICAL AND NUMERICAL RESULTS}

\section{A. Boundary condition}

Let us consider a one-dimensional magnetic thin film where the magnetization $\mathbf{m}$ is allowed to vary in $x$ direction. In this system, the magnetic energy density $W$ reads

$$
W=A\left(\partial_{x} \mathbf{m}\right)^{2}-D(x) \mathbf{m} \cdot\left(\hat{\mathbf{y}} \times \partial_{x} \mathbf{m}\right)-M_{S} \mathbf{m} \cdot \mathbf{H},
$$

where $\partial_{x} \equiv \partial / \partial x, A$ is the symmetric exchange constant, $D(x)$ is the DMI constant that is spatially inhomogeneous along the $x$ direction, $\hat{\mathbf{y}}$ is the unit vector along the perpendicular to both spin-wave propagation direction (i.e., $\hat{\mathbf{x}}$ ) and the thickness direction $\hat{\mathbf{z}}, M_{\mathrm{S}}$ is the saturation magnetization, and $\mathbf{H}$ is the external magnetic field applied in the film plane. Here we assume that the only DMI is inhomogeneous by interface engineering. The corresponding equation of motion 
is the Landau-Lifshitz-Gilbert (LLG) equation, given as

$$
\frac{\partial \mathbf{m}}{\partial t}=\frac{\gamma}{M_{\mathrm{S}}} \mathbf{m} \times \frac{\delta W}{\delta \mathbf{m}}+\alpha \mathbf{m} \times \frac{\partial \mathbf{m}}{\partial t},
$$

where $\alpha$ is the damping, $\mathbf{m}=\mathbf{m}_{0}+\delta \mathbf{m}, \mathbf{m}_{0}$ is the positionindependent magnetization, $\delta \mathbf{m}=\left(\mathbf{0}, \mathbf{s}_{\theta}, \mathbf{s}_{\phi}\right)$ is the spin-wave contribution in the spherical coordinate $\left(s_{\theta}^{2} \ll 1, s_{\phi}^{2} \ll 1\right)$, and $\theta$ and $\phi$ are the polar and azimuthal angles, respectively.

Neglecting the damping, we obtain the effective onedimensional Schrödinger equation for spin-wave wave function $\psi\left(=s_{\theta}+i s_{\phi}\right)$ from the LLG equation as

$$
\begin{aligned}
i \hbar \frac{\partial \psi}{\partial t}=\hat{\mathcal{H}} \psi= & {\left[\frac{\hat{p}_{x}^{2}}{2 m^{*}}-\frac{\left(\mathbf{m}_{0} \cdot \hat{\mathbf{y}}\right)}{\hbar} \alpha_{\mathrm{D}}(x) \hat{p}_{x}\right.} \\
& \left.-\frac{\left(\mathbf{m}_{0} \cdot \hat{\mathbf{y}}\right)}{2 i} \frac{\partial \alpha_{\mathrm{D}}(x)}{\partial x}+\gamma \hbar \mu_{0} H\right] \psi,
\end{aligned}
$$

where $\hat{p}_{x}(\equiv-i \hbar \partial / \partial x)$ is the momentum operator, $m^{*}(\equiv$ $\left.\hbar M_{\mathrm{S}} / 4 \gamma A\right)$ is the effective spin-wave mass, $\alpha_{\mathrm{D}}(x) / \hbar(\equiv$ $\left.2 \gamma D(x) / M_{\mathrm{S}}\right)$ is the DM velocity for spin waves, and $\gamma$ is the gyromagnetic ratio. We note that the third term in the bracket of Eq. (3) is essential for $\hat{\mathcal{H}}$ to be a Hermitian operator. For a system with homogeneous DMI [i.e., $\partial \alpha_{\mathrm{D}}(x) / \partial x=0$ ] and in the absence of the external field, Eq. (3) reproduces our previous result [62].

From the continuity of wave function and integration Eq. (3) for $x$, we obtain the boundary conditions for spin-wave wave function $\psi$ at the boundary between two regions (i.e., $D=D_{1}$ for $x<0$ and $D=D_{2}$ for $x \geqslant 0$ ) as

$$
\begin{aligned}
\psi_{1}(x=0) & =\psi_{2}(x=0), \\
\left.\frac{d \psi_{1}}{d x}\right|_{x=0}-\left.\frac{d \psi_{2}}{d x}\right|_{x=0} & =i \frac{\Delta D}{2 A}\left(\mathbf{m}_{0} \cdot \hat{\mathbf{y}}\right) \psi(x=0),
\end{aligned}
$$

where $\Delta D=D_{2}-D_{1}$. We note that the second boundary condition [Eq. (5)] describes the effect of a DMI step on spin waves, originating from the gradient of DMI in $\mathcal{H}$.

In order to verify the boundary conditions to the spin-wave propagation through a DMI step, we consider a plane spin wave and $\mathbf{m}_{0}=\hat{\mathbf{y}}$, the incident $\left(\psi_{I}\right)$ and reflected $\left(\psi_{R}\right)$ waves in the region 1 where $D=D_{1}$, and the transmitted $\left(\psi_{T}\right)$ wave in the region 2 where $D=D_{2}$ are given as

$$
\psi_{I}=I e^{i k_{1} x}, \quad \psi_{R}=A_{0} e^{-i k_{2} x}, \quad \psi_{T}=B_{0} e^{i k_{3} x},
$$

where $I, A_{0}$, and $B_{0}\left(k_{1}, k_{2}\right.$, and $\left.k_{3}\right)$ are the spin-wave amplitudes (wave numbers) of incident, reflected, and transmitted waves, respectively. From the spin-wave dispersion in each region,

$$
\omega=\gamma \mu_{0}\left(J k^{2}-D_{i}^{*} k+H\right),
$$

where $J=2 A / \mu_{0} M_{\mathrm{S}}$ and $D_{i}^{*}=2 D_{i} / \mu_{0} M_{\mathrm{S}}$ of the $i$ th region ( $i=1,2)$, combined with the boundary conditions, we obtain $A_{0} / I$ and $B_{0} / I$, given as

$$
\begin{aligned}
& \frac{A_{0}}{I}=\frac{\sqrt{\left(D_{1}^{*}\right)^{2}+4 J H^{*}}-\sqrt{\left(D_{2}^{*}\right)^{2}+4 J H^{*}}}{\sqrt{\left(D_{1}^{*}\right)^{2}+4 J H^{*}}+\sqrt{\left(D_{2}^{*}\right)^{2}+4 J H^{*}}}, \\
& \frac{B_{0}}{I}=\frac{2 \sqrt{\left(D_{1}^{*}\right)^{2}+4 J H^{*}}}{\sqrt{\left(D_{1}^{*}\right)^{2}+4 J H^{*}}+\sqrt{\left(D_{2}^{*}\right)^{2}+4 J H^{*}}},
\end{aligned}
$$
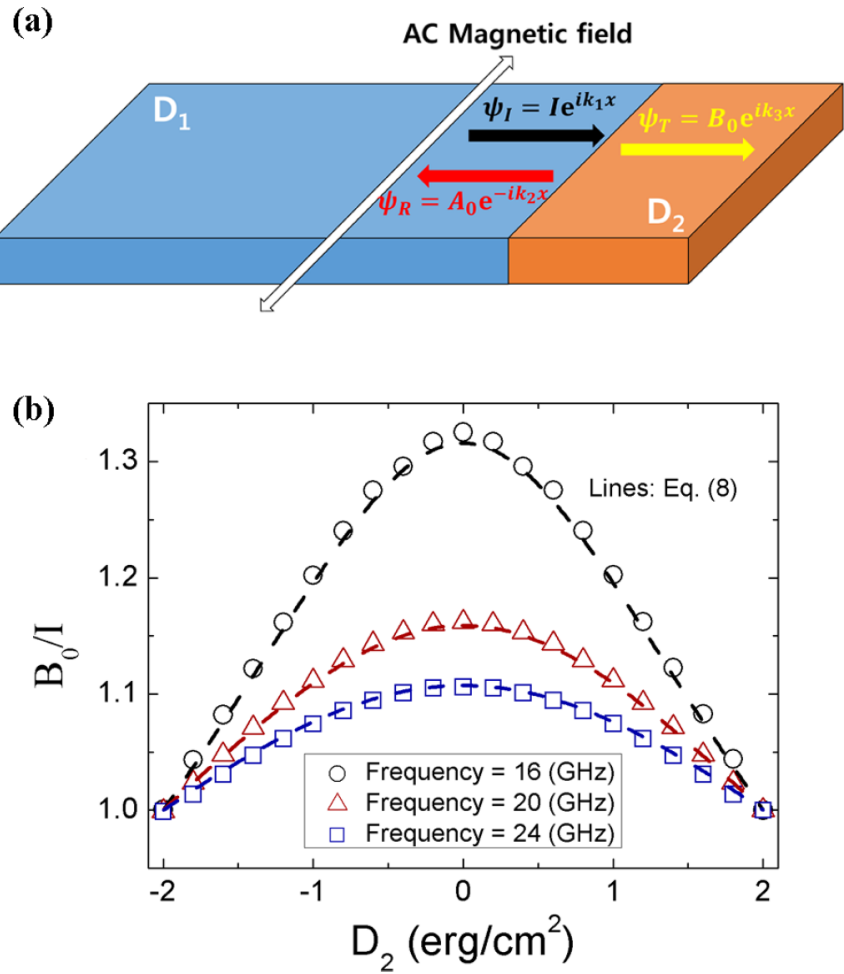

FIG. 1. (a) Coordinate system and schematic illustration of spinwave transmission and reflection at a DMI step. (b) The ratio of transmitted spin wave $\left(B_{0}\right)$ to incident spin wave $(I)$ as a function of $D_{2}$. Parameters: the saturation magnetization $M_{s}=800 \mathrm{kA} / \mathrm{m}$, the exchange stiffness $A=1.3 \times 10^{-11} \mathrm{~J} / \mathrm{m}$, the DMI of the region $1 D_{1}=2 \mathrm{~mJ} / \mathrm{m}^{2}$, the external field $H$ applied along the $y$ axis $=$ $0.5 \mathrm{~T}$, the Gilbert damping $\alpha=0.01$, and the unit cell size along the spin-wave propagation direction $=2 \mathrm{~nm}$.

where $H^{*}=\omega / \gamma \mu_{0}-H$. One finds from Eqs. (8) and (9) that the current conservation holds, i.e., $R+T \equiv 1$, where $R=$ $\left|A_{0} \sqrt{\left|v_{R} / v_{I}\right|} / I\right|^{2}$ and $T=\left|B_{0} \sqrt{\left|v_{T} / v_{I}\right|} / I\right|^{2}$. Here, $v_{I}, v_{R}$, and $v_{T}$ are the group velocities of incident, reflected, and transmitted waves at $x \rightarrow 0$, respectively. The current conservation justifies the boundary conditions. Moreover, Eq. (9) shows $B_{0} / I>1$ when $\left|D_{1}\right|>\left|D_{2}\right|$, i.e., the spin-wave amplification at a DMI step. Numerical simulations based on the LLG equation quantitatively reproduces Eq. (9) (Fig. 1), also justifying the validity of the boundary conditions.

\section{B. Kronig-Penny model}

We next establish a spin-wave version of the Kronig-Penny model. We consider a one-dimensional magnonic crystal with a periodic DMI modulation:

$$
\begin{array}{ll}
\text { Region 1: } D(x)=D_{1}, & n a<x \leqslant(n+1 / 2) a, \\
\text { Region 2: } D(x)=D_{2}, & (n+1 / 2) a<x \leqslant(n+1) a,
\end{array}
$$

where $n=0,1,2, \ldots$, and $a / 2$ is the width of a homogeneous DMI region. Based on the boundary conditions [Eqs. (4) and (5)] and the Bloch's theorem, we obtain an equation for a 
spin-wave version of the Kronig-Penny model as

$$
\begin{aligned}
& \cos \left(k a+\frac{D_{1}^{*}+D_{2}^{*}}{4 J} a \sin \theta\right) \\
& =\cos \frac{a \mu}{2} \cos \frac{a \nu}{2}-\frac{\mu^{2}+v^{2}}{2 \mu \nu} \sin \frac{a \mu}{2} \sin \frac{a \nu}{2},
\end{aligned}
$$

where

$$
\begin{gathered}
\mu=\sqrt{\left(D_{1}^{*} \sin \theta / 2 J\right)^{2}+H^{*} / J}, \\
v=\sqrt{\left(D_{2}^{*} \sin \theta / 2 J\right)^{2}+H^{*} / J} .
\end{gathered}
$$

Here $\theta$ is the angle between the magnetization $\mathbf{m}_{0}$ and the spin-wave propagation direction $\hat{\mathbf{x}}$. Equation (11) allows us to identify the necessary conditions for finite spin-wave band gaps. When $\theta=0$ (i.e., $\mathbf{m}_{0}$ is aligned in the $x$ axis), $\mu$ and $v$ are identical so that the right-hand side of Eq. (11) becomes $\cos (\mu \nu)$; i.e., no spin-wave band gap is expected except for the first forbidden spin-wave band that originates from the external field $H$ and ranges from zero to a finite spin-wave frequency. When $\theta \neq 0$ and $\left|D_{1}\right| \neq\left|D_{2}\right|$, in addition to the first forbidden band, there are always multiple frequency ranges in which the absolute value of the right-hand side of Eq. (11) is greater than the unity. These frequency ranges correspond to the additional spin-wave band gaps.

\section{Tunability of spin-wave band gaps}

In Fig. 2, we summarize the spin-wave forbidden and allowed bands for various parameters. Key features of the spin-wave bands are as follows. The allowed bands become narrower with increasing the lattice constant $a$ [Fig. 2(b)], like results for magnonic crystals constructed with patterned defect structure [40], because the width of the DMI-induced potential barrier for spin waves increases. A similar narrowing of the allowed bands occurs as the angle $\theta$ increases from 0 to $\pi / 2$ [Fig. 2(c)], because the DMI contribution to the spin-wave energy is proportional to $\sin \theta$. For a fixed $D_{1}$, a larger $|\Delta D|$ also results in narrower allowed bands [Fig. 2(d)], because the height of the potential barrier for spin waves increases. The angle variation shown in Fig. 2(c) can be realized by rotating the equilibrium magnetization $\mathbf{m}_{0}$ by means of an external magnetic field. The injection of an in-plane current should also work for this purpose as it generates spin-orbit torque in ferromagnet/heavy metal bilayers $[63,64]$. The DMI variation shown in Fig. 2(d) can be also realized by a local gating [56] that modifies the DMI locally. We note that in all cases, the change in the spin-wave band structure can be very large; e.g., the width of the second forbidden band in Fig. 2(c) varies from $\approx 0 \mathrm{GHz}$ at $\theta=0$ to $5 \mathrm{GHz}$ at $\theta=\pi / 2$. We note that such a large change in the band gap is obtained not only for an abrupt variation of DMI (Fig. 2), but also for a much smoother sinusoidal variation of DMI (not shown). Therefore, one enhances the on/off ratio of spin-wave signals substantially by locating the spin-wave frequency in the frequency ranges in which the band gap varies with $\theta$ or $|\Delta D|$. This efficient tunability of the spin-wave band gap in DMI-modulated magnonic crystals is able to mimic the field-effect transistors in traditional electronics. (a)
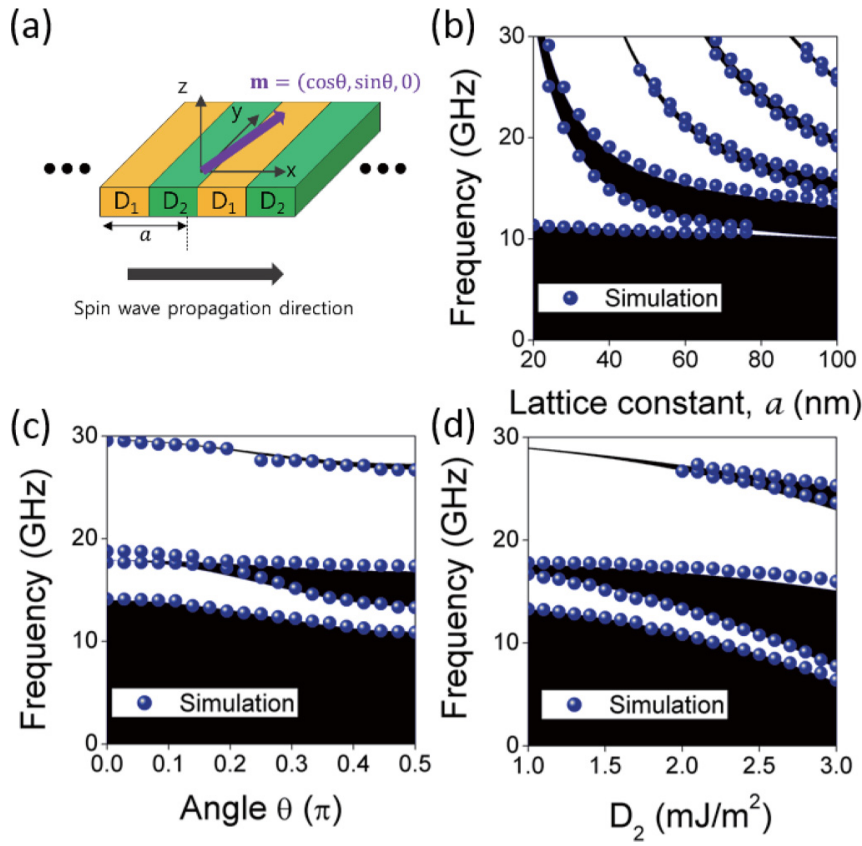

FIG. 2. (a) Coordinate system and schematic illustration of a magnonic crystal with alternating DMI. (b) The spin-wave frequency versus the lattice constant $a$ of an alternating DMI region $(\theta=\pi / 2$, $D_{1}=0 \mathrm{~mJ} / \mathrm{m}^{2}$, and $D_{2}=2 \mathrm{~mJ} / \mathrm{m}^{2}$ ). (c) The spin-wave frequency versus the angle $\theta\left(a=48 \mathrm{~nm}, D_{1}=0 \mathrm{~mJ} / \mathrm{m}^{2}\right.$, and $\left.D_{2}=2 \mathrm{~mJ} / \mathrm{m}^{2}\right)$. (d) The spin-wave frequency versus $D_{2}(a=48 \mathrm{~nm}, \theta=\pi / 2$, and $D_{1}=0 \mathrm{~mJ} / \mathrm{m}^{2}$ ). In (b)-(d), black (white) regions are spin-wave forbidden (allowed) bands calculated from the spin-wave version of the Kronig-Penny model. Circular symbols in (b)-(d) correspond to numerical simulation results. Unless specified, the parameters used for the calculations are the same as in Fig. 1.

In Fig. 2, we also compare the boundaries between the forbidden and allowed bands, obtained from the spin-wave version of the Kronig-Penny model [Eq. (11)], with those obtained by numerically solving the LLG equation (circular symbols). They are in reasonable agreement except for deviations in spin-wave bands as the angle $\theta$ [Fig. 2(c)]. The reason for these deviations is as follows. In the presence of DMI step at $x=i_{0}\left(D=D_{1}\right.$ for $x<i_{0}$ and $D=D_{2}$ for $x \geqslant i_{0}$ ), the DM energy $\mathbf{E}_{\mathrm{DM}, i_{0}}$ is given by

$\frac{\mathbf{E}_{\mathrm{DM}, i_{0}}}{2 \delta x}=D_{1} \hat{\mathbf{y}} \cdot\left(\mathbf{m}_{i_{0}-1} \times \mathbf{m}_{i_{0}}\right)+D_{2} \hat{\mathbf{y}} \cdot\left(\mathbf{m}_{i_{0}} \times \mathbf{m}_{i_{0}+1}\right)$.

Here we substitute $D_{1}$ and $D_{2}$ for $\bar{D}$ and $\Delta D\left(D_{1}=\right.$ $\bar{D}-\Delta D / 2$ and $\left.D_{2}=\bar{D}+\Delta D / 2\right)$; then we obtain the following general equations for the DM energy $E_{\mathrm{DM}}$ and the corresponding DM field $\mathbf{H}_{\mathrm{DM}}$ at the step:

$$
\begin{array}{r}
E_{\mathrm{DM}}=-\bar{D} \mathbf{m} \cdot\left(\hat{\mathbf{y}} \times \frac{\partial \mathbf{m}}{\partial x}\right)-\frac{\Delta D}{2 \delta x} \mathbf{m} \cdot(\hat{\mathbf{y}} \times \overline{\mathbf{m}}), \\
\mathbf{H}_{\mathrm{DM}}=\frac{2}{\mu_{0} M_{\mathrm{S}}}\left(\bar{D} \hat{\mathbf{y}} \times \frac{\partial \mathbf{m}}{\partial x}+\frac{\Delta D}{2 \delta x} \hat{\mathbf{y}} \times \overline{\mathbf{m}}\right),
\end{array}
$$

where $\bar{D}(\overline{\mathbf{m}})$ is the average DMI constant (magnetization) of the nearest neighbor sites crossing the DM step and $\delta x$ is the lattice constant for $\mathbf{m}$. The second term on the right-hand side of Eq. (15) is an additional effective field originating from the 

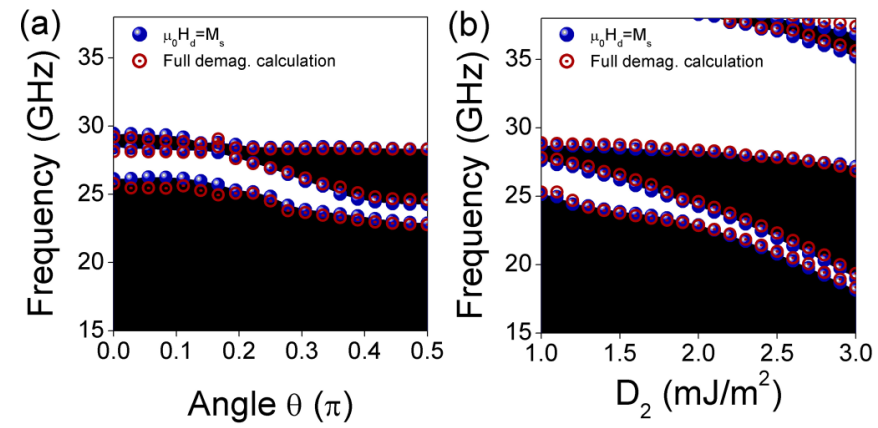

FIG. 3. Spin-wave forbidden and allowed bands in the presence of the demagnetization field $\mu_{0} H_{d}$. (a) The spin-wave frequency versus the angle $\theta\left(a=48 \mathrm{~nm}, D_{1}=0 \mathrm{~mJ} / \mathrm{m}^{2}\right.$, and $\left.D_{2}=2 \mathrm{~mJ} / \mathrm{m}^{2}\right)$. (b) The spin-wave frequency versus $D_{2}(a=48 \mathrm{~nm}, \theta=\pi / 2$, and $D_{1}=0 \mathrm{~mJ} / \mathrm{m}^{2}$ ). In (a) and (b), blue symbols and black (white) regions are spin-wave forbidden (allowed) bands calculated by micromagnetic simulations with $\mu_{0} H_{d}\left(=M_{\mathrm{S}}\right)$. Red symbols in (a) and (b) correspond to simulation results for including full magnetostatic interaction. Unless specified, the parameters used for the calculations are the same as in Fig. 1.

DMI step and acts like a magnetic field applied along the $z$ axis at $\theta=0$ because $\overline{\mathbf{m}} \approx \mathbf{m}_{0}=\hat{\mathbf{x}}$. When $\mathbf{m}_{0}$ deviates from the $y$ axis, this additional effective field tilts the magnetization at the DMI step, causing a deviation of the magnetic state from the uniform state. Therefore, the micromagnetic simulation results have a deviation near $\theta=0$, where the forbidden band is not expected in Eq. (11). As shown in Fig. 2, however, the effect of this additional tilting on the spin-wave band structure is rather weak and does not alter our main conclusion for tunability of the spin-wave band gap.

Finally we show that the efficient tunability of the spin-wave band gap is realized even with a finite demagnetization effect. In the presence of the demagnetization effect, the magnetization undergoes an elliptical precession so that one cannot convert the LLG equation to a Schrödinger-like equation. For this case, therefore, we obtain variations of the spin-wave band structure by numerically solving the LLG equation (Fig. 3). We obtain a qualitatively similar trend to the case with no demagnetization effect (Fig. 2); the allowed bandwidth decreases as either $\theta$ or $|\Delta D|$ (or $D_{2}$ for a fixed $D_{1}$ ) increases. This result confirms that the spin-wave band gap in DMI-modulated magnonic crystals is efficiently tunable regardless of the demagnetization effect. For a comparison, we also plot results for including full magnetostatic interaction (for that purpose, we use the thin film in Fig. 1, discretized along the spin-wave propagation direction. Here the length of the thin film is $4 \mu \mathrm{m}$, the width is $400 \mathrm{~nm}$, and the thickness is $1.5 \mathrm{~nm}$ ). The small deviation of spin-wave band gap in both results is observed but it could be disregarded as anticipated because the film is sufficiently thin.

\section{SUMMARY}

In conclusion, we propose that magnonic crystals with spatially modulated DMI are highly efficient to change the spin-wave band gap by an external means such as a magnetic field, an in-plane current, and a perpendicular voltage gating. This high efficiency is caused by the fact that the DMI contribution to the spin-wave energy is sizable and highly anisotropic depending on the relative orientation of the equilibrium magnetization with respect to the spin-wave propagation direction. We note that the nonlocal magnetostatic interaction, which we ignore in this work, also contributes to the anisotropic dispersion as the spin-wave dispersion of the backward volume mode (i.e., $\mathbf{m}_{0} \| \mathbf{k}$ ) is different from that of the surface mode (i.e., $\mathbf{m}_{0} \perp \mathbf{k}$ ). For an experimentally accessible $D$, however, the DMI contribution is much stronger than the contribution from the magnetostatic interaction [26]. Moreover, the magnetostatic contribution is effective in the small $k$ limit, which makes the scaling of magnonic devices difficult. In contrast, the DMI works for an intermediate to a large $k$ so that magnonic crystals with DMI modulation are expected to be more suitable for higher density devices. The large tunability of spin-wave band gap can enhance the on/off ratio of spin-wave signals as spin waves are unable to propagate when the band gap is large. The proposed magnonic crystals may be useful to reproduce various functionalities of field-effect transistors in traditional electronics at low-power consumption. We end this paper by noting that even though we focus on spin-wave dynamics, our finding will be useful to understand domain wall or skyrmion dynamics in the presence of inhomogeneous DMI as magnetic solitons can be described by spin-wave packets.

\section{ACKNOWLEDGMENTS}

This work was supported by the National Research Foundation of Korea (Grants No. NRF-2015M3D1A1070465, No. NRF2017R1A2B2006119, and No. NRF-2011-0030046) and KUKIST Graduate School of Converging Science and Technology Program.
[1] I. E. Dzyaloshinskii, Sov. Phys. JETP 5, 1259 (1957).

[2] T. Moriya, Phys. Rev. 120, 91 (1960).

[3] U. K. Rößler, A. N. Bogdanov, and C. Pfleiderer, Nature (London) 442, 797 (2006).

[4] M. Uchida, Y. Onose, Y. Matsui, and Y. Tokura, Science 311, 359 (2006).

[5] S. X. Huang and C. L. Chien, Phys. Rev. Lett. 108, 267201 (2012).

[6] A. Fert and P. M. Levy, Phys. Rev. Lett. 44, 1538 (1980).
[7] M. Bode, M. Heide, K. von Bergmann, P. Ferriani, S. Heinze, G. Bihlmayer, A. Kubetzka, O. Pietzsch, S. Blügel, and R. Wiesendanger, Nature (London) 447, 190 (2007).

[8] M. Heide, G. Bihlmayer, and S. Blügel, Phys. Rev. B 78, 140403(R) (2008).

[9] L. Udvardi and L. Szunyogh, Phys. Rev. Lett. 102, 207204 (2009).

[10] Kh. Zakeri, Y. Zhang, T.-H. Chuang, and J. Kirschner, Phys. Rev. Lett. 108, 197205 (2012). 
[11] A. T. Costa, R. B. Muniz, S. Lounis, A. B. Klautau, and D. L. Mills, Phys. Rev. B 82, 014428 (2010).

[12] K.-W. Kim, H.-W. Lee, K.-J. Lee, and M. D. Stiles, Phys. Rev. Lett. 111, 216601 (2013).

[13] A. Thiaville, S. Rohart, É. Jué, V. Cros, and A. Fert, Europhys. Lett. 100, 57002 (2012).

[14] G. Chen, J. Zhu, A. Quesada, J. Li, A. T. N'Diaye, Y. Huo, T. P. Ma, Y. Chen, H. Y. Kwon, C. Won, Z. Q. Qiu, A. K. Schmid, and Y. Z. Wu, Phys. Rev. Lett. 110, 177204 (2013).

[15] S. Emori, U. Bauer, S.-M. Ahn, E. Martinez, and G. S. D. Beach, Nat. Mater. 12, 611 (2013).

[16] K.-S. Ryu, L. Thomas, S.-H. Yang, and S. Parkin, Nat. Nanotechnol. 8, 527 (2013).

[17] S. Mülbauer, B. Binz, F. Jonietz, C. Pfleiderer, A. Rosch, A. Neubauer, R. Georgii, and P. Böni, Science 323, 915 (2009).

[18] X. Z. Yu, Y. Onose, N. Kanazawa, J. H. Park, J. H. Han, Y. Matsui, N. Nagaosa, and Y. Tokura, Nature (London) 465, 901 (2010).

[19] W. Jiang, P. Upadhyaya, W. Zhang, G. Yu, M. B. Jungfleisch, F. Y. Fradin, J. E. Pearson, Y. Tserkovnyak, K. L. Wang, O. Heinonen, S. G. E. te Velthuis, and A. Hoffmann, Science 349, 283 (2015).

[20] S. Woo, K. Litzius, B. Krüger, M.-Y. Im, L. Caretta, K. Richter, M. Mann, A. Krone, R. M. Reeve, M. Weigand, P. Agrawal, I. Lemesh, M.-A. Mawass, P. Fischer, M. Kläui, and G. S. D. Beach, Nat. Mater. 15, 501 (2016).

[21] C. Moreau-Luchaire, C. Moutas, N. Reyren, J. Sampaio, C. A. F. Vaz, N. Van Horne, K. Bouzehouane, K. Garcia, C. Deranlot, P. Warnicke, P. Wohlhter, J.-M. George, M. Weigand, J. Raabe, V. Cros, and A. Fert, Nat. Nanotechnol. 11, 444 (2016).

[22] O. Boulle, J. Vogel, H. Yang, S. Pizzini, D. de S. Chaves, A. Locatelli, T. O. Mentes, A. Sala, L. D. BudaPrejbeanu, O. Klein, M. Belmeguenai, Y. Roussigne, A. Stashkevich, S. M. Cherif, L. Aballe, M. Foerster, M. Chshiev, S. Auffret, I. M. Miron, and G. Gaudin, Nat. Nanotechnol. 11, 449 (2016).

[23] S. D. Pollard, J. A. Garlow, J. Yu, Z. Wang, Y. Zhu, and H. Yang, Nat. Commun. 8, 14761 (2017).

[24] Kh. Zakeri, Y. Zhang, J. Prokop, T.-H. Chuang, N. Sakr, W. X. Tang, and J. Kirschner, Phys. Rev. Lett. 104, 137203 (2010).

[25] D. Cortés-Ortuño and P. Landeros, J. Phys.: Condens. Matter 25, 156001 (2013).

[26] J.-H. Moon, S.-M. Seo, K.-J. Lee, K.-W. Kim, J. Ryu, H.-W. Lee, R. D. McMichael, and M. D. Stiles, Phys. Rev. B 88, 184404 (2013).

[27] K. Di, V. L. Zhang, H. S. Lim, S. C. Ng, M. H. Kuok, J. Yu, J. Yoon, X. Qiu, and H. Yang, Phys. Rev. Lett. 114, 047201 (2015).

[28] H. T. Nembach, J. M. Shaw, M. Weiler, E. Jué, and T. J. Silva, Nat. Phys. 11, 825 (2015).

[29] J. Cho, N.-H. Kim, S. Lee, J.-S. Kim, R. Lavrijsen, A. Solignac, Y. Yin, D.-S. Han, N. J. J. van Hoof, H. J. M. Swagten, and B. Koopmans, Nat. Commun. 6, 7635 (2015).

[30] J. M. Lee, C. Jang, B.-C. Min, S.-W. Lee, K.-J. Lee, and J. Chang, Nano Lett. 16, 62 (2016).

[31] S. Seki, Y. Okamura, K. Kondou, K. Shibata, M. Kubota, R. Takagi, F. Kagawa, M. Kawasaki, G. Tatara, Y. Otani, and Y. Tokura, Phys. Rev. B 93, 235131 (2016).

[32] J.-V. Kim, R. L. Stamps, and R. E. Camley, Phys. Rev. Lett. 117, 197204 (2016).

[33] J. Lan, W. Yu, R. Wu, and J. Xiao, Phys. Rev. X 5, 041049 (2015).
[34] W. Yu, J. Lan, R. Wu, and J. Xiao, Phys. Rev. B 94, 140410(R) (2016).

[35] S. A. Nikitov, Ph. Tailhades, and C. S. Tsai, J. Magn. Magn. Mater. 236, 320 (2001).

[36] A. A. Serga, A. V. Chumak, and B. Hillebrands, J. Phys. D 43, 264002 (2010).

[37] K.-S. Lee, D.-S. Han, and S.-K. Kim, Phys. Rev. Lett. 102, 127202 (2009).

[38] B. Lenk, H. Ulrichs, F. Garbs, and M. Münzenberg, Phys. Rep. 507, 107 (2011).

[39] J. Ding, M. Kostylev, and A. O. Adeyeye, Phys. Rev. Lett. 107, 047205 (2011).

[40] I. Barsukov, F. M. Römer, R. Meckenstock, K. Lenz, J. Lindner, S. Hemken to Krax, A. Banholzer, M. Körner, J. Grebing, J. Fassbender, and M. Farle, Phys. Rev. B 84, 140410(R) (2011).

[41] S. Tacchi, F. Montoncello, M. Madami, G. Gubbiotti, G. Carlotti, L. Giovannini, R. Zivieri, F. Nizzoli, S. Jain, A. O. Adeyeye, and N. Singh, Phys. Rev. Lett. 107, 127204 (2011).

[42] A. D. Karenowska, J. F. Gregg, V. S. Tiberkevich, A. N. Slavin, A. V. Chumak, A. A. Serga, and B. Hillebrands, Phys. Rev. Lett. 108, 015505 (2012).

[43] G. Duerr, K. Thurner, J. Topp, R. Huber, and D. Grundler, Phys. Rev. Lett. 108, 227202 (2012).

[44] A. V. Chumak, V. I. Vasyuchka, A. A. Serga, M. P. Kostylev, V. S. Tiberkevich, and B. Hillebrands, Phys. Rev. Lett. 108, 257207 (2012).

[45] S. Tacchi, G. Duerr, J. W. Klos, M. Madami, S. Neusser, G. Gubbiotti, G. Carlotti, M. Krawczyk, and D. Grundler, Phys. Rev. Lett. 109, 137202 (2012).

[46] M. Krawczyk and D. Grundler, J. Phys.: Condens. Matter 26, 123202 (2014).

[47] A. V. Chumak, V. I. Vasyushka, A. A. Serga, and B. Hillebrands, Nat. Phys. 11, 453 (2015).

[48] M. Mruczkiewicz and M. Krawczyk, Phys. Rev. B 94, 024434 (2016).

[49] K. Vogt, F. Y. Fradin, J. E. Pearson, T. Sebastian, S. D. Bader, B. Hillebrands, A. Hoffmann, and H. Schultheiss, Nat. Commun. 5, 3727 (2014).

[50] M. Vogel, A. V. Chumak, E. H. Waller, T. Langner, V. I. Vasyuchka, B. Hillebrands, and G. von Freymann, Nat. Phys. 11, 487 (2015).

[51] A. Haldar, D. Kumar, and A. O. Adeyeye, Nat. Nanotechnol. 11, 437 (2016).

[52] J. H. Kwon, J. Yoon, P. Deorani, J. M. Lee, J. Sinha, K.-J. Lee, M. Hayashi, and H. Yang, Sci. Adv. 2, e1501892 (2016).

[53] K. Sekiguchi, S.-W. Lee, H. Sukegawa, N. Sato, S.-H. Oh, R. D. McMichael, and K.-J. Lee, NPG Asia Mater. 9, e392 (2017).

[54] A. Khitun, J. Appl. Phys. 111, 054307 (2012).

[55] A. Khitun, J. Appl. Phys. 113, 164503 (2013).

[56] K. Nawaoka, S. Miwa, Y. Shiota, N. Mizuochi, and Y. Suzuki, Appl. Phys. Express 8, 063004 (2015).

[57] G. Chen, T. Ma, A. T. N'Diaye, H. Kwon, C. Won, Y. Wu, and A. K. Schmid, Nat. Commun. 4, 2671 (2013).

[58] J. Torrejon, J. Kim, J. Sinha, S. Mitani, M. Hayashi, M. Yamanouchi, and H. Ohno, Nat. Commun. 5, 4655 (2014).

[59] A. Belabbes, G. Bihlmayer, F. Bechstedt, S. Blügel, and A. Manchon, Phys. Rev. Lett. 117, 247202 (2016).

[60] S. Tacchi, R. E. Troncoso, M. Ahlberg, G. Gubbiotti, M. Madami, J. Akerman, and P. Landeros, Phys. Rev. Lett. 118, 147201 (2017). 
[61] R. de L. Kronig and W. G. Penney, Proc. R. Soc. London A 130, 499 (1931).

[62] A. Manchon, P. B. Ndiaye, J.-H. Moon, H.-W. Lee, and K.-J. Lee, Phys. Rev. B 90, 224403 (2014).
[63] I. M. Miron, K. Garello, G. Gaudin, P.-J. Zermatten, M. V. Costache, S. Auffret, S. Bandiera, B. Rodmacq, A. Schuhl, and P. Gambadella, Nature (London) 476, 189 (2011).

[64] L. Liu, C.-F. Pai, Y. Li, H. W. Tseng, D. C. Ralph, and R. A. Buhrman, Science 336, 555 (2012). 